\title{
Advocacy for Carbomedtherapy (Carbon Dioxide Therapy) in the Treat- ment of Diabetic Neuropathy
}

\author{
Nadia Boudjenah* \\ General Surgeon, Diabetic Foot Surgeon, Diabetic Foot center, Algiers, Algeria
}

*Corresponding author: Nadia Boudjenah, General Surgeon, Diabetic Foot Surgeon, Diabetic Foot center, Algiers, 132 Route Outed Fayet, Amara, Cheraga, Algiers 16003, Algeria, Tel: +213 6618900 17; +33 6815998 00; E-mail: drboudjenah@yahoo.fr

Received: 02 Jul, 2020 | Accepted: 24 Jul, 2020 | Published: 31 Jul, 2020

Citation: Boudjenah N (2020) Advocacy for Carbomedtherapy (Carbon Dioxide Therapy) in the Treatment of Diabetic Neuropathy. Int J Endocrinol Metab Disord 6(2): dx.doi.org/10.16966/2380-548X.169

Copyright: (c) 2020 Boudjenah N. This is an open-access article distributed under the terms of the Creative Commons Attribution License, which permits unrestricted use, distribution, and reproduction in any medium, provided the original author and source are credited.

\section{Introduction}

We are a medical center specializing in the care of the diabetic foot in Algeria for 10 years now. More than $95 \%$ of our patients suffer from severe Neuropathy. Treating Diabetic Neuropathy is a big challenge. More than $50 \%$ of diabetics worldwide are affected. Neuropathy is a consequence of microcirculatory damage in diabetics. The damage caused destroys the nerves. It is caused by the aggression of hyperglycemia [1-4]. The diagnosis is both clinical and paraclinical: ENMG.

Our experience covers more than 60,000 patients, 30\% of whom have no wounds. The clinical examination finds the classic Neuropathy signs: tingling, numbness, cramping, pain, electric shock, loss of especially plantar sensitivity, sensitivity disorder otherwise. In the upper limbs, we look for lesions of the carpal tunnel, as well as lesions of the tarsal canal for the lower limbs. In addition, we note the degree of dryness of hyperkeratosis, the existence of a diabetic foot ulcer or a Charcot foot. Amyotrophy is correlated with the severity of the disorder. It can cause paralysis. We have noticed that the first neurological damage is the Oto-Rhino Laryngitis sphere-we noticed a decrease in hearing. The diastasis recti is a late sign and expresses the severity of Neuropathy.

So, for this fact and to relieve our patients, we first start by stabilizing the diabetes:

- The Hb1Ac should be around 6, which we get easily by enforcing strict diet, hydration, physical activities and above all psychological support - awareness is an important factor.

- We correct the usual Vitamin D3 deficits after dosing.

- We stop all analgesic treatments because we see it as an overwhelming of our patients who reduce considerably their activities, gain weight, unbalance their diabetes to finally worsen their Neuropathy to the point of destroying everything and no longer feeling anything-Paralysis sets in.
- We offer CDT (Carbon Dioxide Therapy) treatment.

\section{What are the Benefits}

- Diet and physical activity help prevent the onset of other complications as well as the obesity related issues, kidney failure, and hypertension.

- The decrease or even disappearance of the reported disorders thereby improving considerably the quality of life.

- The better static balance thereby allowing the return to ambulation, thus increasing theautonomy.

- The CDT sessions allow gradually the recovery of plantar sensitivity, the best prevention of foot wounds, thereby reducing the rate of amputation.

\section{So, What is CDT (Carbon Dioxide Therapy)?}

The carbon dioxide therapy is called Carbomedtherapy for diseases, and carboxytherapy for aesthetics. The CDT consists of transcutaneous injections of carbon dioxide [5-10]. This technic was first practiced, in 1932, by Dr Barrieu, at Royat-Chamalières, France. Initially the indications were purely vascular especially for Raynaud's syndrome [11], arteriopathy of the lower limbs, leg ulcers, and of course, in the care of the diabetic foot.

\section{CDT indications for diabetics}

Diabetic foot: We have two separate tables: 1) Patients with wounds; 2) Patients without wounds, but with vascular or neurological disease. Very often, unfortunately, these aspects are simultaneously entangled.

\section{How to inject?}

We use a device called CDT EVOLUTION. This device delivers heated carbon dioxide during the injections through disposable accessories: $13 \mathrm{~mm}$ and $30 \mathrm{G}$ mesotherapy needles, and sterile tubing. The device being preprogrammed, we use the vascular program, that is to say the one which delivers $80 \mathrm{cc}$ of $\mathrm{CO}_{2}$ per minute. The 
injections are transcutaneous and avoid well the vessels. We inject the surface of the 2 lower limbs, from the Scarpa triangle, around and on the wounds, and in the upper limbs in case of neuropathy. It is an outpatient practice.

The rhythm of the sessions will depend on the severity of the case: once a week, bimonthly, or monthly.

\section{How does it work?}

Hemoglobin molecules have 4 oxygen molecules at saturation. When we inject carbon dioxide, an exchange takes place between these oxygen molecules and those of $\mathrm{CO}_{2}$. This phenomenon is described as BOHR effect [12]. (Figure 1)

We were able to record the instant nature of this exchange, by measuring $\mathrm{PCO}_{2}$ and $\mathrm{PO}_{2}$, during a $\mathrm{CO}_{2}$ injection. What we found was that the $\mathrm{PCO}_{2}$ measurement curve has not been changed while the $\mathrm{PO} 2$ curve recorded an elevation.

\section{What are the Contraindications?}

As a precaution, women who are pregnant. By obligation, patients with an imbalance of tares, especially respiratory and cardiac; Patients who had a recent acute stroke; Patients with active cancers and active viral infections; finally, with anaerobic germ infections. These situations can be reversed, however.

\section{What are the side effects?}

They are benign and above all reversible. There are superficial micro-hematomas at the injection points, which can sometimes follow a session especially if the patient is frankly de-coagulated. They disappear quickly and are not painful. There is no risk of gas embolism. The $\mathrm{CO}_{2}$ pressures delivered by this machine are adjusted to avoid this problem even during accidental injection. The risks of infection should not exist. It is a simple matter of hygiene.

\section{Evolution under CDT treatment}

The evolution under the CDT treatment follows a scheme described below

- In the first place, the tingling disappears then the cramps-we believe that the correction of the Vit D3 rate is not unrelated to this. The pain gradually decreases as the CDT sessions progress. It is imperative to obtain a normal $\mathrm{Hb} 1 \mathrm{Ac}$ level from the patient. Hyperglycemia is a real obstacle to healing by maintaining the perpetual attack of the nerves.

- The second parameter is ambulation, the resumption of which remains difficult when physical activity no longer exists. Exercises performed by a third party to combat stiffness and muscle atrophy are necessary. The use of a wheeled walker allows confidence to be restored for walking autonomy. We take into account the psychological problem linked to the fear that has settled in these patients with multiple histories of falls without counting the effects of the analgesic therapeutic withdrawal that we set up from the start of treatment.

- The recovery of especially plantar sensitivity is done from top to bottom from the Scarpa triangle to the sole of the foot. It manifests itself by the appearance of pain during the injections made during the different sessions.

- For carpal tunnel lesions, disappearance of pain and numbness allowing functional recovery.

- Before starting the CDT treatment sessions, an ENMG (0) of the four limbs is performed by an independent neurologist chosen by the patient. The control is done only after 12 to 24 sessions
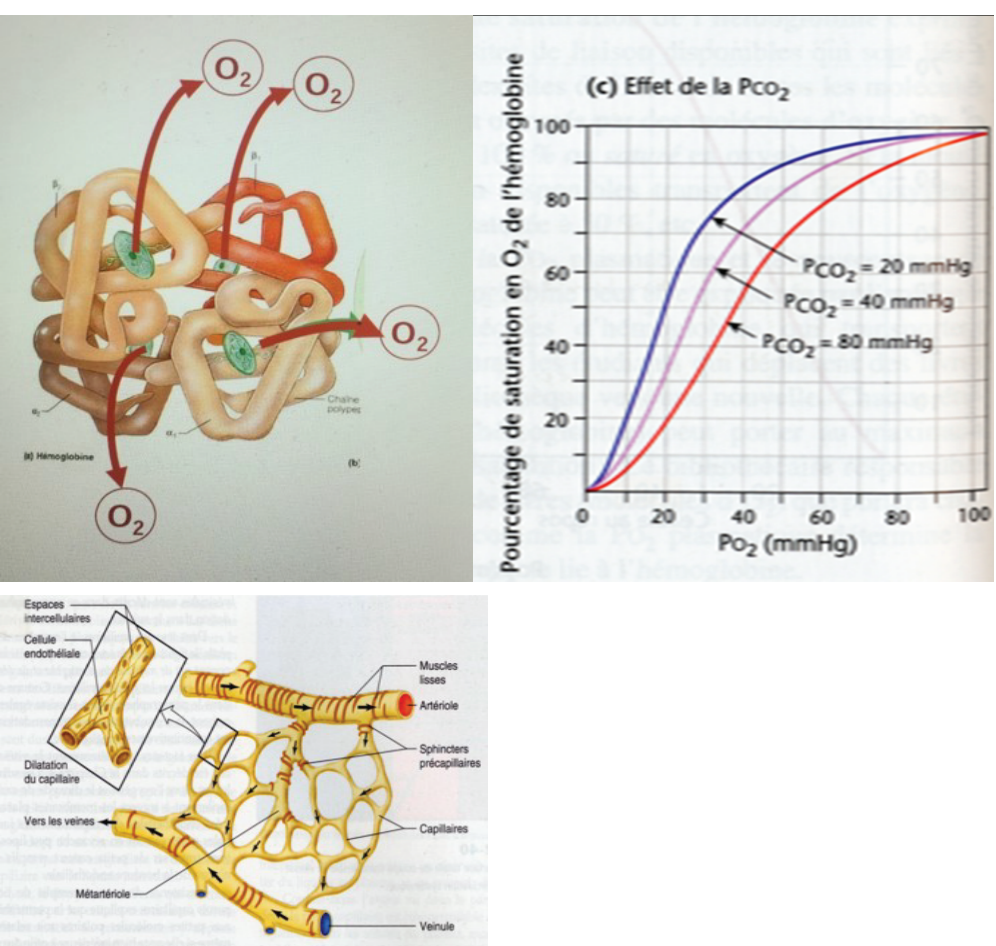

Figure 1: Otherwise, $\mathrm{CO}_{2}$ acts as a vasodilator on the precapillary sphincters, transforming silent zones into functional vascular zones. This is what we call false angiogenesis. This action is perpetuated by the repetition of the sessions by a real angiogenesis. So, there is an influx of blood. At the cellular level, exchanges become more important. 
depending on the severity of the lesions. The sessions' rhythm varies from by-monthly to monthly. The improvement in electrical signs is recorded at one year. The disappearance of Neuropathy in moderate forms without muscle damage is done in one year. In severe forms, 2 to 3 years of treatment are necessary. It is imperative that the level of Hb1Ac stays around 6. The very few failures that we have noted are related to indiscipline and food anarchy. Patients come back regularly because the CDT treatment sessions make the pain disappear. In severe forms with muscle damage, we go through a more or less long stage of stagnation.

Currently, we are preparing 2 studies. First, getting ENMGs' results on a large number of patients. A $2^{\text {nd }}$ study, on the diastasis recti. Our work is unfortunately hampered by the COVID 19 pandemic.

\section{Case Study}

ENMGs below, performed by independent neurologist doctors. We keep all reports for future comparisons.

NEUROLOGY \& NEUROPHYSIOLOGICAL EXPLORATION (EEG, EMC, PE)

\section{CENTER DR. BOUMEDDIENE H.E}

Cite Essalam, 132 logements, BT B, No 11 (RN 04), Khemis Miliana 44225 - Algeria Tel: +213 (0)27 568521 / Cell: +213 (0)55 5611100 dr.boumeddiene@gmail.com

\section{ENMG examination report}

Prescribing doctor: Dr. Nadia Boudjenah

Patient: $\mathbf{x x x x x x x x x x} 75$ years old, Male

Date: Tuesday, November 22, 2016

\section{Description}

- Motor stimulation: Nerves (median, ulnar and distal radial) to upper limbs (UL) objective good motor potential with good amplitude and a lower limit VCM. The F wave is present and of normal latency. The stimulation of the SPE and SPI nerves on both sides finds an alteration of the potential at the Distal stimulation with reduction of the amplitude and collapse of the VCM. The F wave is present and of normal latency.

- Sensitive stimulation: Orthodromic of the anterior and sural tibial nerve finds an alteration of the PAs with reduced VCS, at UL, the sensitive stimulation of the median and ulnar nerve finds a good PAs with conserved VCS.

- Detection: muscles (short thumb abductor, V abductor, PIOD, brachial and deltoid biceps) in UL and anterior and posterior tibial leg and large medial) in the lower limbs (LL) does not find activity at rest with an intermediate line for distal muscles on exertion and an interference pattern for the proximal muscles without signs of active denervation.

Conclusion: ENMG trace to the four limbs in favor of a sensorymotor polyneuropathy essentially symmetrical myelinic and length dependent more marked in the lower limbs. Compatible trace with progressive diabetic neuropathy without signs of active denervation.

\section{Patient: $\operatorname{xxxxxxxxx} 76$ years old, Male}

Date: Sunday, January 07, 2018

Conclusion: ENMG trace in clear improvement compared to the previous trace of 11/22/2016 with significant increase in motor conduction speeds (VCM) to the lower limbs and their- normalization to the upper limbs with increased amplitudes of PAM and PAS. ENMG trace in favor of a moderate diabetic neuropathy in the inferior limbs normal trace in the upper limbs.

\section{Patient: $x x x x x x x x x x 77$ years old, Male}

\section{Date: Monday, September 10, 2018}

Conclusion: ENMG trace objectifying a spectacular improvement in the speeds of motor conduction (remyelinisation) to the lower limbs with a significant gain of the amplitudes but which remain below the norm. The VCS are diminished.

In total: Very good electrical evolution of diabetic neuropathy of the lower limbs with remyelinisation +++ . Normal trace to the upper limbs.

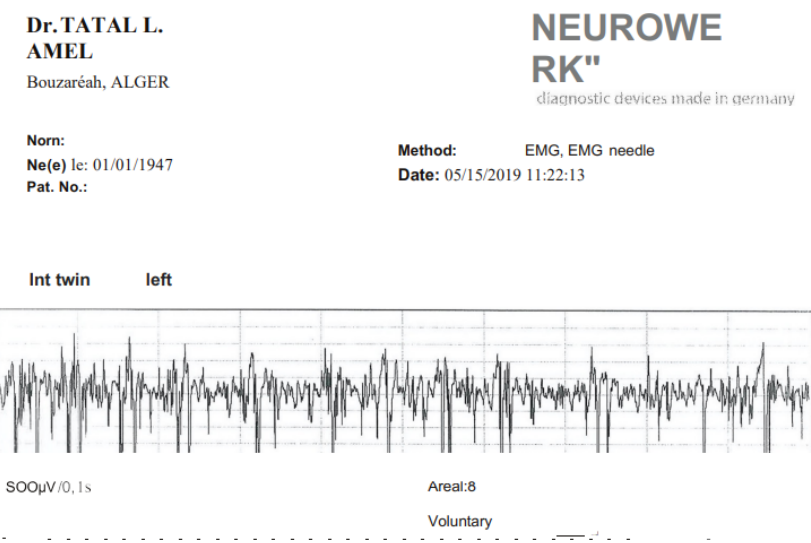

Conclusion: This ENMG exploration of the 04 members exploring the median, ulnar, sensitive and motor fibular tibial and objective $\mathrm{F}$ response: A discreet carpal tunnel on the right of moderate severity. Sensory-motor impairment (poly neuropathy of the axonal type at the level of the 2 lower limbs, sensitivomotor which may be part of a metabolic neuropathy of ancient appearance. Absence of denervation in the resting activity of the anterior leg muscles, twins internal of the 02 dimensions.

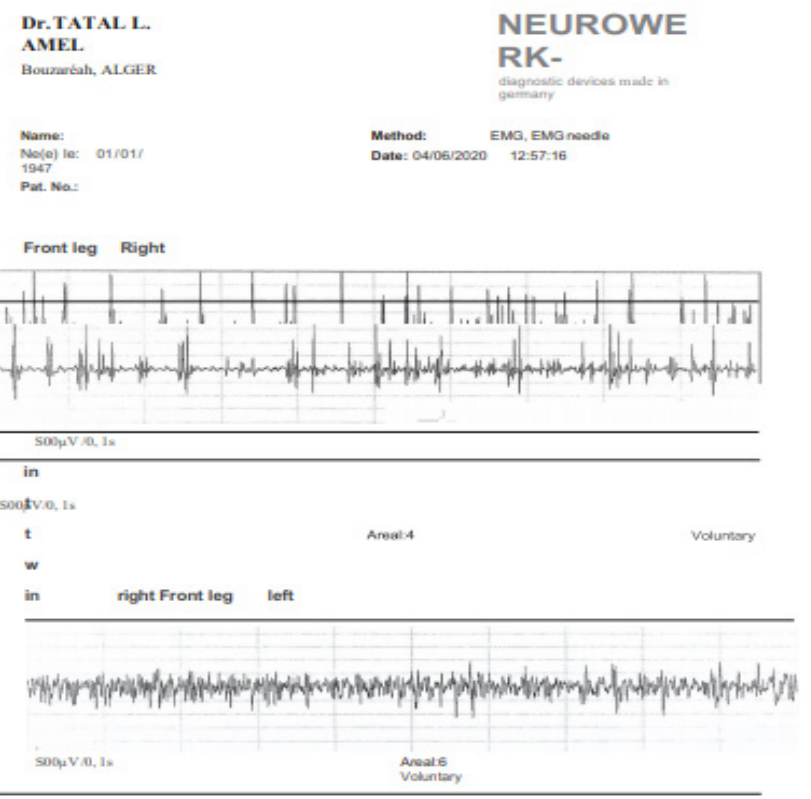


Conclusion: This ENMG exploration of the 02 lower limbs exploring the fibular, tibial sensory and motor nerves, proximal latencies objective an axonal sensitivomotor polyneuropathy of diabetic metabolic origin. There is a marked improvement in amplitudes at the level of the tibial nerves. Needle detection of the anterior leg muscles, internal twins reveals an accelerated intermediate to simple activity but richer in elements compared to the examination of 05/15/2019

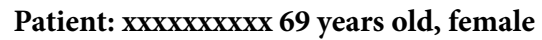

\section{Date: Wednesday, February 13, 2019}

Conclusion: ENMG trace to the four members in favor of:

- Lower limbs: sensory motor neuropathy mainly symmetrical myelinic and length dependent with (reduced VCS and VCM).

- Upper limbs: exclusively symmetrical and distal sensory impairment with (reduced VCS, normal VCM).

- In total: trace compatible with diabetic neuropathy in the 4 limbs, more marked in the lower limbs.

Patient: xxxxxxxxxx 69 years old, female Date: Tuesday, January 14,2020

\section{Conclusion: trace}

ENMG trace to the four limbs in favor of a symmetric distal sensory impairment to the lower limbs with recovery of good motor conduction and correct VCM on both sides. In the upper limbs: ENMG trace without anomalies, sensory motor stimulation is normal on both sides.

In total and compared to the trace of $02 / 13 / 2019$. Very good electrical evolution for lower limbs with normal trace for upper limbs.

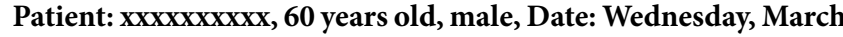
22, 2017

\section{Description}

Motor stimulation: of the median, ulnar and distal radial nerve as well as the SPI on both sides is without anomalies (LDM, amplitude and VCM correct), the F wave is present and of normal latency. The stimulation of the SPE on both sides finds a moderate decrease in the amplitude and decrease in the VCM, the F wave is present.

Sensitive stimulation: orthodromic of the anterior and sural tibial nerve finds a small alteration of the PAs with reduction of the VCS. That of the median and ulnar nerve is normal.

Detection: muscles (short adductor of the thumb, PIOD, brachial and deltoid biceps at UL and anterior and tibial leg posterior to LL) do not find spontaneous activity at rest with an interference trace proximally, intermediate distally and LL without signs of denervation.

Conclusion: ENMG trace to the four members in favor of a moderate sensory motor polyneuropathy localized to the lower limbs (LL) and the anterior compartment without signs of active denervation. (Beginner diabetic neuropathy). The hind compartment of the lower limbs (LL) and the upper limbs (UL) are unharmed.

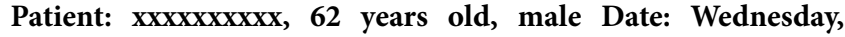
January 02, 2019

\section{Conclusion}

- Trace ENMG to the four limbs in favor of a pure demyelinating moderate sensory impairment symmetrical to the lower limbs.
- Favorable evolution compared to the trace of $22 / 03 / 17$ on the motor plan with recovery of correct VCM (SPE G from $25 \mathrm{~m} / \mathrm{s}$ to $35.9 \mathrm{~m} / \mathrm{s}$, SPE D from $36.1 \mathrm{~m} / \mathrm{s}$ to $41.3 \mathrm{~m} / \mathrm{s}$ ).

- Persistence of moderate sensory impairment in the lower limbs.

- Trace normal to the upper limbs.

\section{Cabinet De Neurologie Et De Neurophysiologie EEG/PSMNIEMG/PES/PEMIPEAIPEVIERGIPEA/ASSR Dr. Dib Khaled \\ Cite Segna Bt 01 Reghaia Alger \\ Tel/ Fax: +213 21.84.06.40}

\section{EMG Report}

Prescribing doctor: Dr. Nadia Boudjenah

Name: XXXXXXXX Age: 74 years old

female Date: 01/07/2017

Clinical information: Follow-up for Diabetic Neuropathy.

ATCDS: since 1980 (30 years).

Neurography: Study of the median nerve, ulnar, SPE, SPIand sural. Upper limbs

- Motor latencies of the median and ulnar nerve, normal amplitudes and $\mathrm{vcm}$

- Absent sensory potential of the median and lunar nerve

- $\quad$ F wave on the median nerve, absent.

Lower limbs

SPE and SPI matrix latencies with normal VCM, reduced amplitude

- $\quad$ Absent sensory potential of the sural nerve.

- $\quad \mathrm{F}$ wave on the SPI nerve absent.

- $\quad H$ reflexes on the tibial nerve: weak amplitude.

Conclusion: The EMG exploration to date reveals the persistence of electrophysiological signs in favor of a diffuse sensory motor neuropathy of essentially sensitive axonal type, probably diabetic.

\section{Name: XXXXXXXX, Age: 75 years old}

female Date: 09/22/2018

Clinical information: Diabetes checkup.

Neurography: Study of the median nerve, ulnar, SPE, SPIand ddc. Upper limbs

- Motor latencies of the median and ulnar nerve, normal amplitudes and $\mathrm{vcm}$.

- Sensory latencies of the median and ulnar nerve, normal amplitude and vcs.

- $\quad \mathrm{F}$ wave on the median nerve of normal latency.

Lower limbs

SPE and SPI matrix latencies with normal vcm, reduced amplitude

- Absent sensory potential of the sural nerve.

- $\quad$ F wave on the SPI nerve absent.

- $\quad H$ reflexes on the tibial nerve: present. 
Conclusion: The EMG exploration to date reveals the persistence of electrophysiological signs in favor of a diffuse sensory motor neuropathy of essentially sensitive axonal type, probably diabetic.

\section{Conclusion}

Little by little, a good number of our patients saw a significant decrease and even disappearance of the reported disorders of the Neuropathy, considerably improving their quality of life. From the wheelchair when they arrived first time at our center, the better static balance recovery with CDT treatments, allowed them to return to ambulation thereby increasing their autonomy. CDT is a simple and inexpensive technique that currently seems, to us, to be the only therapeutic alternative in the treatment of Diabetic Neuropathy. We have several studies underway and will publish them in the near future.

\section{References}

1. Cameron NE, Eaton SE, Cotter MA, Tesfaye S (2001) Vascular factors and metabolic interactions in the pathogenesis of Diabetic neuropathy. Diabetologia 44: 1973-1988.

2. Cameron NE, Cotter MA (1997) Metabolic and vascular factors in the pathogenesis of diabetic neuropathy. Diabetes 46: S31-S37.

3. Ward JD (1995) Biochemical and vascular factorsin the pathogenesis of diabetic neuropathy. Clin Invest Med 18: 267-274.
4. Hartemann A, Lozeron P (2015) Les Neuropathies Périphériques chez les Diabétiques. Janvier-Février 74 MCED: 70-73.

5. Boudjenah N (2020) Diabetic Foot in Algeria. Int J Endocrinol Metab Disord 6.

6. Boudjenah N (2016) Introduction of Carbomedtherapy. Endocrine Practice. Pro Quest 22.

7. Brandi C, Grimaldi L, Nisi G, Brafa A, Campa A, et al. (2010) The Role of Carbon Dioxide Therapy in the Treatment of Chronic Wounds. In Vivo 24: 223-236.

8. Nisi G, Barberi L, Ceccaccio L, Cuomo R, Sisti A, et al. (2015) Effect of repeated subcutaneous injections of carbon dioxide $\left(\mathrm{CO}_{2}\right)$ on inflammation linked to hypoxia inadipose tissue graft. Eur Rev Med Pharmacol Sci 19: 4501-4506.

9. Kovacevic J, Sinozic T (2014) Carboxytherapy: supportive therapy in wound management. Société française dePhlébologie 67: 46-52.

10. Khiat L, Leibaschoff GH (2018) Clinical prospective study on the use of subcutaneous carboxytherapy in the treatment of diabeticfoot ulcer. Surg Technol Int 32: 81-90.

11. Schmidt J, Monnet P, Normand B, Fabry R (2005) Microcirculatory and clinical effects of serial percutaneous application of carbondioxide in primary and secondary Raynaud's phenomenon. Vasa 34: 93-100.

12. Sakai Y, Miwa M, Oe K, Ueha T, Koh A, et al. (2011) A novel system for transcutaneous application of carbon dioxide causing an "artificial Bohr effect" in the human body. PLoS One 6: e24137. 